\title{
A Note on Three Approaches to Connexivity
}

\author{
Ricardo Arturo Nicolás-Francisco ${ }^{1}$ (])
}

'Graduate Program in Philosophy of Science, National Autonomous University of Mexico (UNAM), Mexico

ORCID: R.A.N.F. 0000-0001-5490-9357

\section{Sorumlu yazar/Corresponding author:} Ricardo Arturo Nicolás-Francisco, Graduate Program in Philosophy of Science, National Autonomous University of Mexico (UNAM), Mexico

E-mail/E-posta: gyl.ric@gmail.com

Başvuru/Submitted: 11.11.2019

Kabul/Accepted: 10.12.2019

\section{Atıf/Citation:}

Nicolás-Francisco, Ricardo Arturo. (2019). "A Note on Three Approaches to Connexivity" Felsefe Arkivi-Archives of Philosophy, 51: 129138. https://doi.org/10.26650/arcp2019-5108

\section{ABSTRACT}

In this paper I explore whether three different approaches to connexivity - namely, Lewis and Langford's, Wansing's and Egré and Politzer's - can be non-trivially related. Lewis and Langford's approach consists of the definition of the conditional in terms of consistency via the definition of possibility in terms of consistency and Lewis' definition of the (strict) conditional in terms of possibility; Wansing's approach is based on his definition of the falsity condition for the conditional as "if $A$ is true then $B$ is false"; Egré and Politzer's approach relies on the modalization of the falsity condition for the conditional in its conditional form $(A \rightarrow \diamond \sim B)$.

With this background in mind, I build upon Estrada-González's work relating connexivism and possibilism, the thesis according to which everything is possible. Estrada-González's work relates Lewis and Langford's connexive approach with Nelson's idea that every proposition is self-consistent. He does so using a fragment of LP augmented with a special conditional. This relation has resulted in the study and postulation of several truth-functional modalities, some of which have already been discussed elsewhere. I employ several truth-functional modalities to evaluate Egré and Politzer's falsity conditions for the conditional. I show that in using Béziau's truth-functional modality $\diamond_{B^{\prime}}$ definable in Estrada-González's framework, one can retain in a single framework the three different approaches. More specifically, distinguishing the notion of logical equivalence from the notion of interderivability and these two from the notion of coimplication, it is possible to relate $\sim(A \rightarrow B)$ and $(A \rightarrow \diamond \sim B)$ as interderivable schemas and in a valid biconditional. I also show some consequences of this for Béziau's negative appraisal of three-valued truthfunctional modalities.

Keywords: Possibilism, connexivism, conditional, consistency, modalities 


\section{Introduction}

Connexive logics are logics in which the following schemas are valid:

(Aristotle's theses)

$$
\begin{aligned}
& \sim(A \rightarrow \sim A), \sim(\sim A \rightarrow A) \\
& (A \rightarrow B) \rightarrow \sim(A \rightarrow \sim B),(A \rightarrow \sim B) \rightarrow \sim(A \rightarrow B)
\end{aligned}
$$

(Boethius' theses)

Whereas the following is invalid:

$$
\text { (Symmetry of implication) } \quad(A \rightarrow B) \rightarrow(B \rightarrow A)
$$

In a recent article, Hitoshi Omori [6] brings into relation two views of connexive logics. One is taken from Heinrich Wansing's definition of the falsity condition for the conditional [7] -the condition that tells under what situation the conditional is false- and the other from Paul Egré and Guy Politzer's modalization of the falsity condition for the conditional [2].

Wansing considers that the falsity condition for the conditional is "If $A$ is true then $B$ is false" rather than the standard condition " $A$ is true and $B$ is false". Paul Egré and Guy Politzer, on the other hand, propose two modalized schemas $-(A \wedge \diamond-B)$ and $(A \rightarrow \diamond-B)-$ as schemas equivalent to $\sim(A \rightarrow B)$, and called them 'weak forms' in order to distinguish them from the more common schemas $(A \wedge \sim B)$ and $(A \rightarrow \sim B)$ - what they called 'strong forms'.

Omori brings into relation those views by considering that the falsity condition for the conditional is the modalized schema $(A \rightarrow \diamond \sim B)$. Under certain conditions, Wansing's approach results in a limit case of Egré and Politzer's.

In a different vein, Luis Estrada-González [3] has related connexivism and possibilism by probing a view on consistency from Everett Nelson's idea of (self-)consistency [5], and a view on possibility from C. I. Lewis and C. H. Langford's definition of possibility [4].

Lewis and Langford defined possibility in terms of consistency, $\diamond(A \wedge B)={ }_{\text {def }} A \mathrm{o} B$, and, through Lewis' definition of the (strict) conditional in terms of possibility, $(A \rightarrow B)=_{\text {def }}$ $\checkmark \nabla(A \wedge \sim B)$, the conditional in terms of consistency, $(A \rightarrow B)=_{\text {def }}(A 0-B)$. Nelson, in turn, proposed that every proposition is self-consistent preserving the definition of the conditional in terms of consistency, but rejected the inter-definability of consistency and possibility.

Estrada-González draws a connection between Nelson's and Lewis and Langford's proposals using a fragment of González-Asenjo/Priest's logic LP augmented with a conditional named 'CO' ('CO' stands for Cantwell-Omori). The connection of those views resulted in the distinction of several truth-functional modalities. 
Omori has demonstrated that any logic that satisfies

$$
\begin{aligned}
& (A \rightarrow A) \\
& (A \leftrightarrow \sim \sim A) \\
& ((A \rightarrow A) \rightarrow B) \leftrightarrow B \\
& (B \rightarrow C) \rightarrow((A \rightarrow B) \rightarrow(A \rightarrow C)) \\
& \frac{(A \rightarrow B), A}{B} \\
& \frac{(A \rightarrow B)}{(\diamond A \rightarrow \diamond B)}
\end{aligned}
$$

Plus Wansing's falsity condition for the conditional

$$
\sim(A \rightarrow B) \leftrightarrow(A \rightarrow \sim B)
$$

and Paul Egré and Guy Politzer’s form,

$$
\sim(A \rightarrow B) \leftrightarrow(A \rightarrow \diamond \sim B)
$$

such logic has $(\diamond B \leftrightarrow B)$ as a valid schema. It means that if one adds $\diamond B$ as a valid schema, equivalent to $\sim(B \rightarrow \sim B)$, Aristotle's Thesis in Lewis and Langford's setting, one trivializes the logic. As a consequence, it is not possible to have Wansing's, Egré and Politzer's, Lewis and Langford's, and Nelson's approaches to connexive logic in one non-trivial framework.

In this paper I will show a simple model in which it is possible to retain Wansing's, Egré and Politzer's, and Lewis and Langford's approaches, which has also some consequences for some negative appraisals of three-valued truth-functional modalities, like that of Béziau in [1]. The structure of the paper is as follows. In Section 2 I present some technical preliminaries. Then, in Section 3 I present the evaluation conditions for the different modalities and the evaluation of the modalized falsity conditions for the conditional. Finally, in Section 4 I discuss some outcomes of this evaluation.

\section{Preliminaries}

Consider a language $L$ with a denumerable set $P R O P=\left\{p_{1}, p_{2}, p_{3} \ldots\right\}$ of propositional variables and with the connectives $\rightarrow$ and $\sim$. The notion of a formula is defined in the usual way. Capital letters $A, B$, etc., are used as variables for formulas. I will use ' $F O R M$ ' to denote the set of formulas of $L$.

Let $V=\left\{T,{ }^{*}, F\right\}$ be a set of truth values, ordered by $F^{* *}<T$, with $D^{+}=\left\{T,{ }^{*}\right\}$ and $D^{-}=\{F\}$ as the set of designated and anti-designated values, respectively. A function $v: P R O P \rightarrow V$ is 
an assignment of truth values to propositional variables that can be extended to all formulas satisfying the conditions shown in the following truth-tables:

\begin{tabular}{|c|c|c|c|}
\hline$A$ & $-A$ & $B$ & $A \rightarrow B$ \\
\hline$T$ & $F$ & $T$ & $T$ \\
\hline$T$ & $F$ & $*$ & $*$ \\
\hline$T$ & $F$ & $F$ & $F$ \\
\hline$*$ & $*$ & $T$ & $T$ \\
\hline$*$ & $*$ & $*$ & $*$ \\
\hline$*$ & $*$ & $F$ & $F$ \\
\hline$F$ & $T$ & $T$ & $*$ \\
\hline$F$ & $T$ & $*$ & $*$ \\
\hline$F$ & $T$ & $F$ & $*$ \\
\hline
\end{tabular}

That is, the value of the conditional is the value for the consequent if the antecedent has a designated value. Otherwise, it has the value *. Negation is as in LP, namely:

\begin{tabular}{|c|c|}
\hline$A$ & $-A$ \\
\hline$T$ & $F$ \\
\hline$*$ & $*$ \\
\hline$F$ & $T$ \\
\hline
\end{tabular}

A second negation, ᄀ, stronger than $\sim$, can be introduced:

\begin{tabular}{|c|c|}
\hline$A$ & $A\urcorner$ \\
\hline$T$ & $F$ \\
\hline$*$ & $F$ \\
\hline$F$ & $T$ \\
\hline
\end{tabular}

Following the distinctions of levels of theoremhood made by Estrada-González, I will call 'p-theorems' those formulas that are never antidesignated; 'T-theorems' those formulas that are always designated; 'supertheorems' those schemas that are always designated and at least once (just) true; and 'q-theorems' those schemas that are always just true.

$A$ and $B$ are equivalent if and only if they have the same truth values under any assignment. $A$ and $B$ are inter-derivable if and only if whenever one is the premise the other is the conclusion in a valid argument. $A$ and $B$ are coimplicative of each other if and only if $(A \rightarrow B)$ and $(B \rightarrow A)$ are always designated.

\section{Just a few modalities}

All the distinct modalities considered in the following are defined as negations of conditionals with schemas implying its own negation, as it is indicated under each modality. The lower index attached to each modality distinguishes the kind of modality that will be employed. The evaluation conditions for each modality are the result of evaluating its respective conditionals and negations. 


\begin{tabular}{|c|c|c|c|c|}
\cline { 2 - 5 } \multicolumn{1}{c|}{} & $\diamond_{\mathrm{L}} A$ & $-\diamond_{\mathrm{L}} A$ & $\diamond_{\mathrm{L}} \sim A$ & $-\diamond_{\mathrm{L}} \sim A$ \\
\hline$A$ & $-(A \rightarrow \sim A)$ & $\sim-(A \rightarrow-A)$ & $-(\sim A \rightarrow \sim A)$ & $\sim-(\neg A \rightarrow \sim A)$ \\
\hline$T$ & $T$ & $F$ & $*$ & $*$ \\
\hline$*$ & $*$ & $*$ & $*$ & $*$ \\
\hline$F$ & $*$ & $*$ & $T$ & $F$ \\
\hline
\end{tabular}

These are Lewis and Langford's modalities as evaluated in this three-valued setting. It is easy to see that any formula of the form $\diamond_{\mathrm{L}} A$ is designated under any assignment, hence the relation with possibilism. The next ones employ rather the strong negation:

\begin{tabular}{|c|c|c|c|c|}
\cline { 2 - 5 } \multicolumn{1}{c|}{} & $\diamond_{\mathrm{Lc}} A$ & $\neg \diamond_{\mathrm{Lc}} A$ & $\diamond_{\mathrm{Lc}} \neg A$ & $\neg \diamond_{\mathrm{Lc}} \neg A$ \\
\hline$A$ & $\neg(A \rightarrow \neg A)$ & $\neg \neg(A \rightarrow \neg A)$ & $\neg(\neg A \rightarrow \neg \neg A)$ & $\neg \neg(\neg A \rightarrow \neg \neg A)$ \\
\hline$T$ & $T$ & $F$ & $F$ & $T$ \\
\hline$*$ & $T$ & $F$ & $F$ & $T$ \\
\hline$F$ & $F$ & $T$ & $T$ & $F$ \\
\hline
\end{tabular}

The modalities below are introduced by Béziau in [1], and they are obtained by mixing both negations in non-possibility, impossibility and necessity:

\begin{tabular}{|c|c|c|c|c|}
\cline { 2 - 5 } \multicolumn{1}{c|}{} & $\diamond_{\mathrm{B}} A$ & $-\diamond_{\mathrm{B}} A$ & $\diamond_{\mathrm{B}} \neg A$ & $\sim \diamond_{\mathrm{B}} \sim A$ \\
\hline$A$ & $\neg(A \rightarrow \neg A)$ & $\neg(A \rightarrow \neg A)$ & $\neg(\neg A \rightarrow \neg \sim A)$ & $\sim \neg(\neg A \rightarrow \neg \neg A)$ \\
\hline$T$ & $T$ & $F$ & $F$ & $T$ \\
\hline$*$ & $T$ & $F$ & $T$ & $F$ \\
\hline$F$ & $F$ & $T$ & $T$ & $F$ \\
\hline
\end{tabular}

These are also obtained by mixing both negations in non-possibility, impossibility and necessity, but starting with possibility defined with $\sim$ :

\begin{tabular}{|c|c|c|c|c|}
\cline { 2 - 5 } \multicolumn{1}{c|}{} & $\diamond_{\mathrm{L}} A$ & $\neg \diamond_{\mathrm{L}} A$ & $\diamond_{\mathrm{L}} \neg A$ & $\neg \diamond_{\mathrm{L}} \neg A$ \\
\hline$A$ & $\sim(A \rightarrow \neg A)$ & $\neg \sim(A \rightarrow \neg A)$ & $\sim(\neg A \rightarrow \sim \neg A)$ & $\neg \sim(\neg A \rightarrow \sim \neg A)$ \\
\hline$T$ & $T$ & $F$ & $*$ & $F$ \\
\hline$*$ & $*$ & $F$ & $*$ & $F$ \\
\hline$F$ & $*$ & $F$ & $T$ & $F$ \\
\hline
\end{tabular}

The next two groups of modalities are obtained by mixing the two negations already since the definition of possibility:

\begin{tabular}{|c|c|c|c|c|}
\cline { 2 - 5 } \multicolumn{1}{c|}{} & $\diamond_{\mathrm{M} 1} A$ & $\sim \diamond_{\mathrm{M} 1} A$ & $\diamond_{\mathrm{M} 1} \sim A$ & $\sim \diamond_{\mathrm{M} 1}-A$ \\
\hline$A$ & $\sim(A \rightarrow \neg A)$ & $\sim \sim(A \rightarrow \neg A)$ & $\sim(\sim A \rightarrow \neg \sim A)$ & $\sim \sim(\neg A \rightarrow \neg-A)$ \\
\hline$T$ & $T$ & $F$ & $*$ & $*$ \\
\hline$*$ & $T$ & $F$ & $T$ & $F$ \\
\hline$F$ & $*$ & $*$ & $T$ & $F$ \\
\hline
\end{tabular}




\begin{tabular}{|c|c|c|c|c|}
\cline { 2 - 5 } \multicolumn{1}{c|}{} & $\diamond_{\mathrm{M1}} A$ & $\neg \diamond_{\mathrm{M1}} A$ & $\diamond_{\mathrm{M} 1} \neg A$ & $\neg \diamond_{\mathrm{M} 1} \neg A$ \\
\hline $\mathrm{A}$ & $\sim(A \rightarrow \neg A)$ & $\neg \sim(A \rightarrow \neg A)$ & $\sim(\neg A \rightarrow \neg \neg A)$ & $\neg \sim(\neg A \rightarrow \neg \neg A)$ \\
\hline$T$ & $T$ & $F$ & $*$ & $F$ \\
\hline$*$ & $T$ & $F$ & $*$ & $F$ \\
\hline$F$ & $*$ & $F$ & $T$ & $F$ \\
\hline
\end{tabular}

The last two groups of modalities are identified by Estrada-González, but not discussed in detail. I just make explicit their evaluation conditions. As it can be easily checked, they collapse in the present setting based on LP, although they could come apart in an FDE-framework.

\begin{tabular}{|c|c|c|c|c|}
\cline { 2 - 5 } \multicolumn{1}{c|}{} & $\diamond_{\mathrm{M} 2} A$ & $-\diamond_{\mathrm{M} 2} A$ & $\diamond_{\mathrm{M} 2} \sim A$ & $\sim \diamond_{\mathrm{M} 2} \sim A$ \\
\hline$A$ & $\neg(A \rightarrow \sim A)$ & $\neg(A \rightarrow \neg A)$ & $\neg(\neg A \rightarrow \sim \neg A)$ & $\sim \neg(\neg A \rightarrow \sim-A)$ \\
\hline$T$ & $T$ & $F$ & $F$ & $T$ \\
\hline$*$ & $F$ & $T$ & $F$ & $T$ \\
\hline$F$ & $F$ & $T$ & $T$ & $F$ \\
\hline
\end{tabular}

\begin{tabular}{|c|c|c|c|c|}
\cline { 2 - 5 } \multicolumn{1}{c|}{} & $\diamond_{\mathrm{M} 2} A$ & $\neg \diamond_{\mathrm{M} 2} A$ & $\diamond_{\mathrm{M} 2} \neg A$ & $\neg{ }$ \\
\hline $\mathrm{A}$ & $\neg(A \rightarrow \neg A)$ & $\neg \neg(A \rightarrow \sim A)$ & $\neg(\neg A \rightarrow \sim \neg A)$ & $\neg \neg(A \rightarrow \sim \neg A)$ \\
\hline$T$ & $T$ & $F$ & $F$ & $T$ \\
\hline$*$ & $F$ & $T$ & $F$ & $T$ \\
\hline$F$ & $F$ & $T$ & $T$ & $F$ \\
\hline
\end{tabular}

\section{The connection}

Now I proceed to evaluate Wansing's and Egré and Politzer's falsity conditions for the conditional and make some comments on the results: I will do so first, with the help of the truth values without modalities, and then with each modality.

\begin{tabular}{|c|c|c|c|c|c|c|c|}
\hline$A$ & $B$ & $-B$ & $A \rightarrow B$ & $A \wedge \sim B$ & $A \rightarrow \sim B$ & $\sim(A \rightarrow B)$ & $\neg(A \rightarrow B)$ \\
\hline$T$ & $T$ & $F$ & $T$ & $F$ & $F$ & $F$ & $F$ \\
\hline$T$ & $*$ & $*$ & $*$ & $*$ & $*$ & $*$ & $F$ \\
\hline$T$ & $F$ & $T$ & $F$ & $T$ & $T$ & $T$ & $T$ \\
\hline$*$ & $T$ & $F$ & $T$ & $F$ & $F$ & $F$ & $F$ \\
\hline$*$ & $*$ & $*$ & $*$ & $*$ & $*$ & $*$ & $F$ \\
\hline$*$ & $F$ & $T$ & $F$ & $*$ & $T$ & $T$ & $T$ \\
\hline$F$ & $T$ & $F$ & $*$ & $F$ & $*$ & $*$ & $F$ \\
\hline$F$ & $*$ & $*$ & $*$ & $F$ & $*$ & $*$ & $F$ \\
\hline$F$ & $F$ & $T$ & $*$ & $F$ & $*$ & $*$ & $F$ \\
\hline
\end{tabular}

It is easy to verify that $A \rightarrow \sim B$ and $\sim(A \rightarrow B)$ are equivalent in this case. Now, I turn to the modalities: 


\begin{tabular}{|c|c|c|c|c|c|c|c|}
\hline$A$ & $B$ & $\left.\diamond_{\mathrm{Lc}}\right\urcorner B$ & $A \rightarrow B$ & $A \wedge \diamond_{\mathrm{Lc}} \neg B$ & $A \rightarrow \diamond_{\mathrm{Lc}} \neg B$ & $-(A \rightarrow B)$ & $\neg(A \rightarrow B)$ \\
\hline$T$ & $T$ & $F$ & $T$ & $F$ & $F$ & $F$ & $F$ \\
\hline$T$ & $*$ & $F$ & $*$ & $F$ & $F$ & $*$ & $F$ \\
\hline$T$ & $F$ & $T$ & $F$ & $T$ & $T$ & $T$ & $T$ \\
\hline$*$ & $T$ & $F$ & $T$ & $F$ & $F$ & $F$ & $F$ \\
\hline$*$ & $*$ & $F$ & $*$ & $F$ & $F$ & $*$ & $F$ \\
\hline$*$ & $F$ & $T$ & $F$ & $*$ & $T$ & $T$ & $T$ \\
\hline$F$ & $T$ & $F$ & $*$ & $F$ & $*$ & $*$ & $F$ \\
\hline$F$ & $*$ & $F$ & $*$ & $F$ & $*$ & $*$ & $F$ \\
\hline$F$ & $F$ & $T$ & $*$ & $F$ & $*$ & $*$ & $F$ \\
\hline
\end{tabular}

\begin{tabular}{|c|c|c|c|c|c|c|c|}
\hline$A$ & $B$ & $\left.\diamond_{\mathrm{M} 2}\right\urcorner B$ & $A \rightarrow B$ & $\left.A \wedge \diamond_{\mathrm{M} 2}\right\urcorner$ & $\left.A \rightarrow \diamond_{\mathrm{M} 2}\right\urcorner$ & $\sim(A \rightarrow B)$ & $\neg(A \rightarrow B)$ \\
\hline$T$ & $T$ & $F$ & $T$ & $F$ & $F$ & $F$ & $F$ \\
\hline$T$ & $*$ & $F$ & $*$ & $F$ & $F$ & $*$ & $F$ \\
\hline$T$ & $F$ & $T$ & $F$ & $T$ & $T$ & $T$ & $T$ \\
\hline$*$ & $T$ & $F$ & $T$ & $F$ & $F$ & $F$ & $F$ \\
\hline$*$ & $*$ & $F$ & $*$ & $F$ & $F$ & $*$ & $F$ \\
\hline$*$ & $F$ & $T$ & $F$ & $*$ & $T$ & $T$ & $T$ \\
\hline$F$ & $T$ & $F$ & $*$ & $F$ & $*$ & $*$ & $F$ \\
\hline$F$ & $*$ & $F$ & $*$ & $F$ & $*$ & $*$ & $F$ \\
\hline$F$ & $F$ & $T$ & $*$ & $F$ & $*$ & $*$ & $F$ \\
\hline
\end{tabular}

Even though $\sim(A \rightarrow B)$ and $\left(A \wedge \diamond_{\mathrm{Lc}} \neg B\right)$ are not equivalent (nor they are $\sim(A \rightarrow B)$ and $\left.\left(A \rightarrow \diamond_{\mathrm{M} 2} \neg B\right)\right)$, there is an interesting interaction between $\neg(A \rightarrow B)$ and $\left(A \wedge \diamond_{\mathrm{Lc}} \neg B\right)$, and also between $\left(A \wedge \diamond_{\mathrm{M} 2} \neg B\right)$ and $\neg(A \rightarrow B)$. They are not equivalent because they differ in the assignation of one value (sixth row). However, in both cases that value is designated. If we define a logical consequence relation as preservation of designated values (from premises to conclusion $)^{1}$, then $\neg(A \rightarrow B)$ implies $\left(A \wedge \diamond_{\mathrm{Lc}} \neg B\right)$ and vice versa. They are inter-derivable. In this sense, it relates the (strong) falsity condition for the conditional with a (weak) conjunctive version of it.

\begin{tabular}{|c|c|c|c|c|c|c|c|}
\hline$A$ & $B$ & $\diamond_{\mathrm{B}} \sim B$ & $A \rightarrow B$ & $A \wedge \diamond_{\mathrm{B}}-B$ & $A \rightarrow \diamond_{\mathrm{B}} \sim B$ & $\sim(A \rightarrow B)$ & $\neg(A \rightarrow B)$ \\
\hline$T$ & $T$ & $F$ & $T$ & $F$ & $F$ & $F$ & $F$ \\
\hline$T$ & $*$ & $T$ & $*$ & $T$ & $T$ & $*$ & $F$ \\
\hline$T$ & $F$ & $T$ & $F$ & $T$ & $T$ & $T$ & $T$ \\
\hline$*$ & $T$ & $F$ & $T$ & $F$ & $F$ & $F$ & $F$ \\
\hline$*$ & $*$ & $T$ & $*$ & $*$ & $T$ & $*$ & $F$ \\
\hline$*$ & $F$ & $T$ & $F$ & $*$ & $T$ & $T$ & $T$ \\
\hline$F$ & $T$ & $F$ & $*$ & $F$ & $*$ & $*$ & $F$ \\
\hline$F$ & $*$ & $T$ & $*$ & $F$ & $*$ & $*$ & $F$ \\
\hline$F$ & $F$ & $T$ & $*$ & $F$ & $*$ & $*$ & $F$ \\
\hline
\end{tabular}

1 Where capital Greek letters $\Gamma, \Sigma, \Delta$ denote sets of formulas, I write ' $F A$ ' if and only if there is no assignment that makes all the formulas in $\Gamma$ designated and $A$ not designated. 
Similar to the previous cases, the schemas $\left(A \rightarrow \diamond_{\mathrm{B}}-B\right)$ and $\sim(A \rightarrow B)$ are not equivalent because they differ in two values (second and fifth rows), but in both cases those values are designated, so they are inter-derivable. Moreover, they are coimplicative, because $\left(A \rightarrow \diamond_{\mathrm{B}} \sim B\right) \rightarrow \sim(A \rightarrow B)$ and $\sim(A \rightarrow B) \rightarrow\left(A \rightarrow \diamond_{\mathrm{B}} \sim B\right)$ are always valid, as the reader can easily verify.

The subsequent modalities are the ugliest:

\begin{tabular}{|c|c|c|c|c|c|c|c|}
\hline$A$ & $B$ & $\diamond_{\mathrm{L}}-B$ & $A \rightarrow B$ & $A \wedge \diamond_{\mathrm{L}}-B$ & $A \rightarrow \diamond_{\mathrm{L}}-B$ & $\sim(A \rightarrow B)$ & $\neg(A \rightarrow B)$ \\
\hline$T$ & $T$ & $*$ & $T$ & ${ }^{*}$ & ${ }^{*}$ & $F$ & $F$ \\
\hline$T$ & $*$ & $*$ & $*$ & $*$ & ${ }^{*}$ & $*$ & $F$ \\
\hline$T$ & $F$ & $T$ & $F$ & $T$ & $T$ & $T$ & $T$ \\
\hline$*$ & $T$ & $*$ & $T$ & $*$ & ${ }^{*}$ & $F$ & $F$ \\
\hline$*$ & $*$ & $*$ & $*$ & $*$ & $*$ & $*$ & $F$ \\
\hline$*$ & $F$ & $T$ & $F$ & $*$ & $T$ & $T$ & $T$ \\
\hline$F$ & $T$ & $*$ & $*$ & $F$ & ${ }^{*}$ & $*$ & $F$ \\
\hline$F$ & $*$ & $*$ & $*$ & $F$ & $*$ & $*$ & $F$ \\
\hline$F$ & $F$ & $T$ & $*$ & $F$ & $*$ & $*$ & $F$ \\
\hline
\end{tabular}

\begin{tabular}{|c|c|c|c|c|c|c|c|}
\hline$A$ & $B$ & $\diamond_{\perp} \neg B$ & $A \rightarrow B$ & $A \wedge \diamond_{\perp} \neg B$ & $A \rightarrow \diamond_{\perp} \neg B$ & $\sim(A \rightarrow B)$ & $\neg(A \rightarrow B)$ \\
\hline$T$ & $T$ & $*$ & $T$ & $*$ & ${ }^{*}$ & $F$ & $F$ \\
\hline$T$ & $*$ & $*$ & $*$ & $*$ & ${ }^{*}$ & $*$ & $F$ \\
\hline$T$ & $F$ & $T$ & $F$ & $T$ & $T$ & $T$ & $T$ \\
\hline$*$ & $T$ & $*$ & $T$ & $*$ & $*$ & $F$ & $F$ \\
\hline$*$ & $*$ & $*$ & $*$ & $*$ & $*$ & $*$ & $F$ \\
\hline$*$ & $F$ & $T$ & $F$ & $*$ & $T$ & $T$ & $T$ \\
\hline$F$ & $T$ & $*$ & $*$ & $F$ & $*$ & $*$ & $F$ \\
\hline$F$ & $*$ & $*$ & $*$ & $F$ & $*$ & $*$ & $F$ \\
\hline$F$ & $F$ & $T$ & $*$ & $F$ & $*$ & $*$ & $F$ \\
\hline
\end{tabular}

\begin{tabular}{|c|c|c|c|c|c|c|c|}
\hline$A$ & $B$ & $\diamond_{\mathrm{M} 1}-B$ & $A \rightarrow B$ & $A \wedge \diamond_{\mathrm{M} 1}-B$ & $A \rightarrow \diamond_{\mathrm{M} 1}-B$ & $\sim(A \rightarrow B)$ & $\neg(A \rightarrow B)$ \\
\hline$T$ & $T$ & $*$ & $T$ & $*$ & ${ }^{*}$ & $F$ & $F$ \\
\hline$T$ & $*$ & $T$ & $*$ & $T$ & $T$ & $*$ & $F$ \\
\hline$T$ & $F$ & $T$ & $F$ & $T$ & $T$ & $T$ & $T$ \\
\hline$*$ & $T$ & $*$ & $T$ & $*$ & $*$ & $F$ & $F$ \\
\hline$*$ & $*$ & $T$ & $*$ & $*$ & $T$ & $*$ & $F$ \\
\hline$*$ & $F$ & $T$ & $F$ & $*$ & $T$ & $T$ & $T$ \\
\hline$F$ & $T$ & $*$ & $*$ & $F$ & $*$ & $*$ & $F$ \\
\hline$F$ & $*$ & $T$ & $*$ & $F$ & $*$ & $*$ & $F$ \\
\hline$F$ & $F$ & $T$ & $*$ & $F$ & $*$ & $*$ & $F$ \\
\hline
\end{tabular}




\begin{tabular}{|c|c|c|c|c|c|c|c|}
\hline$A$ & $B$ & $\diamond_{\mathrm{M} 1} \neg B$ & $A \rightarrow B$ & $\left.A \wedge \diamond_{\mathrm{M} 1}\right\urcorner B$ & $\left.A \rightarrow \diamond_{\mathrm{M} 1}\right\urcorner B$ & $-(A \rightarrow B)$ & $\neg(A \rightarrow B)$ \\
\hline$T$ & $T$ & $*$ & $T$ & $*$ & ${ }^{*}$ & $F$ & $F$ \\
\hline$T$ & $*$ & $*$ & $*$ & $*$ & ${ }^{*}$ & $*$ & $F$ \\
\hline$T$ & $F$ & $T$ & $F$ & $T$ & $T$ & $T$ & $T$ \\
\hline$*$ & $T$ & $*$ & $T$ & $*$ & $*$ & $F$ & $F$ \\
\hline$*$ & $*$ & $*$ & $*$ & $*$ & $*$ & $*$ & $F$ \\
\hline$*$ & $F$ & $T$ & $F$ & $*$ & $T$ & $T$ & $T$ \\
\hline$F$ & $T$ & $*$ & $*$ & $F$ & $*$ & $*$ & $F$ \\
\hline$F$ & $*$ & $*$ & $*$ & $F$ & $*$ & $*$ & $F$ \\
\hline$F$ & $F$ & $T$ & $*$ & $F$ & $*$ & $*$ & $F$ \\
\hline
\end{tabular}

These are some salient facts that can be easily verified with the truth tables:

- Any formula of the form $A \rightarrow \diamond-B$ is a supertheorem with the modalities $\diamond_{\mathrm{L}^{-}}, \diamond_{\mathrm{L}} \neg, \diamond_{\mathrm{M} 1} \sim$ and $\left.\diamond_{\mathrm{M} 1}\right\urcorner$.

- There is no collapse of the two falsity conditions for the conditional under any modality.

- Any formulas of the form $\left(A \wedge \diamond_{\mathrm{L}} \neg B\right)$ and $\left(A \wedge \diamond_{\mathrm{L}}-B\right)$ are equivalent.

- Any formulas of the form $\left(A \rightarrow \diamond_{\mathrm{L}} \neg B\right)$ and $\left(A \rightarrow \diamond_{\mathrm{L}}-B\right)$ are equivalent.

$-\neg(A \rightarrow B)$ is not equivalent to $(A \wedge \diamond \sim B)$ nor to $(A \rightarrow \diamond \sim B)$ under any modality.

$-\sim(A \rightarrow B)$ is not equivalent to $(A \wedge \diamond \sim B)$ nor to $(A \rightarrow \diamond \sim B)$ under any modality.

However, we have seen that there are modalities for which $\neg(A \rightarrow B)$ implies $(A \wedge \diamond \neg B)$ and vice versa, namely $\diamond_{\mathrm{Lc}}$ and $\diamond_{\mathrm{M} 2}$. Similarly, there is a modality for which $\sim(A \rightarrow B)$ implies $(A \rightarrow \diamond-B)$ and vice versa, namely $\diamond_{\mathrm{B}}$.

$\diamond_{\mathrm{B}}$, is, then, the modality that can relate the three approaches to connexivity. It is interesting to note that this was the modality that, according to Béziau [1], was considered by Lukasiewicz in a system where nothing is necessary but not everything is possible. Béziau considers the following truth-table $(s)^{2}$ for necessity, $\square$, with $D^{+}=\{T\}$ and $D^{-}=\{*, F\}$ :

\begin{tabular}{|c|c|c|}
\hline$\square A$ & $A$ & $\diamond A$ \\
\hline$D$ & $F$ & $D^{-}$ \\
\hline$D^{-}$ & $*$ & $T$ \\
\hline$D^{-}$ & $T$ & $T$ \\
\hline
\end{tabular}

That is, necessity can have just non-designated values. Béziau claims that in a three-valued logic with two non-designated values, necessity can have just non-designated values, for in this way one could deal with a basic modal logic, i.e., a logic in which the following axioms are valid:

$\square A \vdash A \quad A \vdash \diamond A$

$\square A \nvdash A \quad \diamond A \nvdash A$

2 This is not a truth-table, but a set of truth-tables, as the values in the set of non-designated values can be precisified in several ways. 
However, in Estrada-González’s setting, Béziau's necessity -defined as $\square_{B}=_{\text {def }} \sim \diamond_{B} \sim-$ is at least true in one case, as the following table shows

\begin{tabular}{|c|c|c|}
\hline$\square_{\mathrm{B}} A$ & $A$ & $\diamond_{\mathrm{B}} A$ \\
\hline$D^{-}$ & $F$ & $D^{-}$ \\
\hline$D^{-}$ & $*$ & $T$ \\
\hline$T$ & $T$ & $T$ \\
\hline
\end{tabular}

This assignment for necessity has nothing to do with the election of the set of designated and nondesignated values (in Beziau's view, the election of two designated values amounts to the assignment of only designated values for possibility, which is not even the case in Estrada-González's setting).

I think that the distribution of the truth-values in Estrada-Gonzalez's truth-table is the most natural, for if the distribution of values for possibility is the result of the following condition:

$v\left(\diamond_{\mathrm{B}} A\right)=F$ (or non-designated) if and only if $v(A)=F$, and $v\left(\diamond_{\mathrm{B}} A\right)=T$ in all other cases

one would expect the distribution of values for necessity to be the result of the dual condition: $v\left(\diamond_{\mathrm{B}} A\right)=T$ if and only if $v(A)=T$, and $v\left(\diamond_{\mathrm{B}} A\right)=F$ (or non-designated) in all other cases.

\section{Conclusions}

After presenting the falsity conditions for the conditional à la Egré-Politzer, and the modalities in Estrada-González's paper, I have proceeded to evaluate the falsity conditions with those modalities. The result is that the three approaches of connexivity - Lewis and Langford's, Wansing's and Egré and Politzer's approaches - are related via the modality $\diamond_{\mathrm{B}}: \sim(A \rightarrow B)$ and $(A \rightarrow \diamond \sim B)$ are inter-derivable.

Acknowledgments: This paper was written under the support of the PAPIIT project IN403719 "Intensionality all the way down: a new plan for logical relevance". I want to thank Luis Estrada-González for discussion, helpful comments and suggestions on some previous versions of this paper.

\section{References}

Béziau, J.-Y. "A new four-valued approach to modal logic", Logique et Analyse 213, no. 54 (2011): 109-121.

Egré, P. and Politzer, G. "On the negation of indicative conditionals", In Proceedings of the Amsterdam Colloquium, edited by M. Franke, M. Aloni and F. Roelofsen, 10-18, 2013.

Estrada-González, L. "Possibility, consistency, connexivity", unpublished typescript, (2019).

Lewis, C. I. and Langford, C. H. Symbolic Logic, New York: Dover Publications, 1932.

Nelson, E. "Intensional relations" Mind 156, no. 39 (1930): 440-453.

Omori, H. "Towards a bridge over two approaches in connexive logic" Logic and Logical Philosophy, no. 28 (2019): 553-566.

Wansing, H. "Connexive modal logic" In Advances in Modal Logic, vol. 5 edited by R. Schmidt, I. PrattHartmann, M. Reynolds and H. Wansing, 367-383. London: King's College Publications, 2005. 